\title{
Effectiveness of occlusion therapy in ametropic amblyopia: a pilot study
}

\author{
Merrick J Moseley, Alistair R Fielder, Mary Irwin, Helen S Jones, Rosemary J Auld
}

\begin{abstract}
Academic Unit of Ophthalmology, Imperial College of Science, Technology and Medicine, London M J Moseley A R Fielder
\end{abstract}

Academic Unit of Ophthalmology, The University of Birmingham, Birmingham M Irwin

H S Jones

Department of Orthoptics, The Birmingham and Midland Eye Centre, Birmingham

R J Auld

Correspondence to: Dr M J Moseley, Academic Unit of Ophthalmology, The Western Eye Hospital, Marylebone Road, London NW1 5YE.

Accepted for publication 6 June 1997

\begin{abstract}
Aims/background-To examine the relative contributions of non-specific (for example, spectacle correction) and specific (that is, occlusion therapy) treatment effects on children with ametropic amblyopia. To assess the importance and practicality of objectively confirming the prescribed occlusion dose.

Methods-Subjects were entered into a two phase trial. In the first ('pretreatment') subjects were provided with spectacle correction and underwent repeat visual acuity (VA) and contrast sensitivity (CS) testing until acuity in their amblyopic eye had stabilised. Subjects then progressed to the second phase ('treatment') in which they underwent direct, unilateral occlusion for 1 hour per day for 4 weeks. Patching was objectively monitored using an occlusion dose monitor.

Results-Eight subjects completed the trial, all but one of whom achieved $>80 \%$ concordance with the occlusion regimen. Within the pretreatment phase, mean amblyopic eye VA improved by $0.19 \mathrm{log}$ units $(p=0.008)$ while mean CS gained 0.09 $\log$ units $(p=0.01)$. An identical improvement in mean VA was recorded in the fellow eyes $(p=0.03)$ while mean $C S$ gained $0.11 \log$ units $(p=0.02)$. Within the treatment phase, mean VA further improved (0.12 log units, $p=0.009)$ although this gain had halved by the end of treatment and was no longer statistically significant $(\mathrm{p}=0.09)$.
\end{abstract}

Conclusions-Visual performance improved significantly during pretreatment whereas further gains seen during occlusion were not sustained. Evaluation of occlusion regimens must take into consideration the potentially confounding influence of 'pretreatment effects' and the necessity to confirm objectively the occlusion dose a child receives.

(Br f Ophthalmol 1997;81:956-961)

It is widely believed that patching the better seeing eye of a child with amblyopia can improve spatial resolution in the affected eye. This observation - mainly based on clinical experience rather than empirical determination-disguises the fact that treatment effectiveness has not been unequivocally established. ${ }^{1}$ Unsurprisingly, widely differing treatment regimens have been promoted; some practitioners favouring full time ${ }^{2}$ while others just a few minutes' occlusion each day. ${ }^{3}$ Such a diversity of opinion serves only to highlight that the amount of occlusion necessary to improve vision is quite unknown and, as a result, recommendations for vague, pragmatically derived occlusion regimens have proliferated within paediatric ophthalmic texts. ${ }^{45}$

While accepting that occlusion is beneficial, there are sound reasons for adopting a general therapeutic principle - namely, to prescribe the smallest occlusion dose to bring about an effective treatment response. This point is cogently illustrated by a recent study which analysed the outcome of 342 amblyopic children who underwent occlusion in a routine clinical setting. ${ }^{6}$ Prescribed occlusion dose ranged from 14 to 3167 hours: $33 \%,<99$ hours; 18\%, 100-199 hours; 20\%, 200-499 hours; and $28 \%$, more than 500 hours. The value of a treatment which makes such demands on patient and family must be questioned when only $19 \%$ of those in the highest dose band attained an acuity of 6/9.

Our failure to have determined the effectiveness of occlusion is due, at least in part, to the difficulty of recording, with any useful degree of precision, the amount of occlusion a child actually receives. Concordance (formally 'compliance" ${ }^{7}$ ) with patching is known to be highly variable,${ }^{89}$ probably dose dependent ${ }^{19}$ and is held to be one of the most important factors influencing outcome. ${ }^{10-12}$ Yet, until recently the only quantitative and objective measure of concordance has been that of clinic attendance which clearly does not properly constitute a measure of occlusion dose.

An additional complexity is the need to ensure that gains in visual performance are solely attributable to the regimen under evaluation rather than to other non-specific treatment effects. For example, it is well known that children may take time to adapt to spectacle correction worn before occlusion; indeed this intervention alone is considered by some to be an effective treatment in its own right. ${ }^{13}{ }^{14}$ Also, a child may not optimally perform visual tasks until they are familiar with the tester and test environment, something which might not occur within the time constraints of a single session.

In this pilot study we sought insight into the problems posed both by concordance and possible confounding of specific by non-specific treatment effects. We did not investigate the effects of aetiology, age, severity, or differing occlusion regimens, which remain to be examined in future randomised controlled trials. 
All prescribed occlusion was objectively recorded using an occlusion dose monitor (ODM).$^{15}$ Briefly, this device records patchskin contact by bioelectric means providing a measure, not only of the total duration of occlusion (dose), but also of that occurring on a daily basis (regimen, dose rate). To identify benefits solely attributable to occlusion we did not begin patching immediately upon diagnosis. Subjects underwent a period of 'pretreatment' in which they were provided with the appropriate spectacle correction and their visual performance monitored at intervals of 2 weeks until visual acuity in the amblyopic eye had stabilised.

We chose to employ a single occlusion regimen: direct, unilateral patching for 1 hour per day for a duration of 4 weeks. Minimal occlusion regimens have been reported to bring about a therapeutic response, ${ }^{3}$ while minimising the burden placed upon child and parent.

\section{Patients and methods}

Subjects were recruited from children attending the Birmingham and Midland Eye Hospital having been referred from either a community screening service or from their family practitioners. Visual acuity was recorded using an internally illuminated, logarithmically scaled (logMAR) chart (Lighthouse Low Vision Products, Cat No C110) and letter contrast sensitivity using a Pelli-Robson chart (Clement Clarke Ltd). The acuity chart was viewed at 4 metres and scored on a letter by letter basis giving a resolution of $0.02 \mathrm{log}$ units. ${ }^{16}$ The contrast sensitivity chart was viewed at 1 metre and also scored on a letter by letter basis providing a resolution of $0.05 \mathrm{log}$ units. ${ }^{17}$ Each subject then underwent a full ophthalmic examination including cycloplegic retinoscopy and autorefraction. Spectacles were prescribed but specific instructions were given to parents for these not to be worn until the next assessment.

Inclusion criteria were: a diagnosis of ametropic amblyopia without strabismus or other ocular pathology; no prior history of occlusion therapy or other orthoptic treatment; and an interocular difference in visual acuity of at least $0.1 \log$ units.

The study protocol had been approved by the district ethics committee of West Birmingham Area Health Authority. Written consent to participate in the trial was obtained from the subjects' parents all of whom were provided with an information sheet describing the study.

The experimental protocol comprised two consecutive phases, the first ('pretreatment') beginning approximately 14 days after subjects' initial examination and enrolment (Fig 1). On entering this phase the previously prescribed spectacles were worn for the first time. Corrected acuity and letter contrast sensitivity were recorded and subjects scheduled to return in 14 days. Spectacle wear alone was permitted and no occlusion was prescribed at this time. Assessments continued at 14 day intervals until any improvement (defined as a gain of $0.02 \mathrm{log}$ units or greater) in the visual acuity of the amblyopic eye had ceased. At this point subjects progressed to the second ('treatment') phase of the trial in which they were prescribed 4 weeks of direct, unilateral occlusion for 1 hour per day. This treatment was objectively monitored using an $\mathrm{ODM}^{15}-\mathrm{a}$ small battery powered datalogger. This device, worn either attached to a belt or carried in a shoulder bag connects via a lead concealed under clothing to an eye patch modified by the addition of two miniature electrodes to its undersurface. By continuously recording the resistance across the patch electrodes the ODM maintains an occlusion time history which can, at intervals, be down loaded to a PC for storage and analysis of concordance. Parents were also asked to complete an occlusion diary indicating the time at which occlusion began and ended on each day of the treatment phase.

While undergoing occlusion therapy, subjects returned at weekly intervals for visual performance assessment (undertaken throughout the trial by a single examiner) and an appraisal of the objective occlusion record. Feedback was provided to parents as to the extent of treatment concordance and, where necessary, encouragement given to comply with the treatment regimen. At the end of the treatment phase, subjects' clinical status was reviewed and, where indicated, appropriate clinical interventions continued in a routine setting.

The principal analysis compared the changes in acuity and contrast sensitivity that occurred: within the pretreatment phase (that is, at start and week of maximum improvement) between pretreatment and during treatment phases (that is, maximum pretreatment scores versus maximum treatment scores) and, between pretreatment and end of treatment phases (that is, maximum pretreatment scores versus end of treatment scores).

Significance of change was assessed using related samples $t$ tests.

\section{Results}

Of the 10 subjects initially enrolled, one attained near normal acuity in both eyes during pretreatment (interocular difference $\leqslant 0.1 \mathrm{log}$ units). We chose to exclude this subject from further involvement in the trial on the basis that the occlusion regimen could not be considered clinically appropriate for the mildness of their condition. A second subject was mistakenly entered into the treatment phase before vision in their amblyopic eye had stabilised and on this basis their data are excluded from the analysis presented here.

The remaining two male and six female subjects had mean (SD) age of 5.1 (1.9) years. Amblyopia was associated with anisometropia in five of the children, with bilateral hypermetropic and astigmatic refractive errors in two children and, in the remaining child, with a bilateral astigmatic error. Full subject details appear in Table 1.

Pretreatment lasted from 2 to 16 weeks (median 6 weeks). At the start of this phase the mean corrected visual acuity of subjects' amblyopic eyes was $0.61 \log$ MAR (6/24) with a 


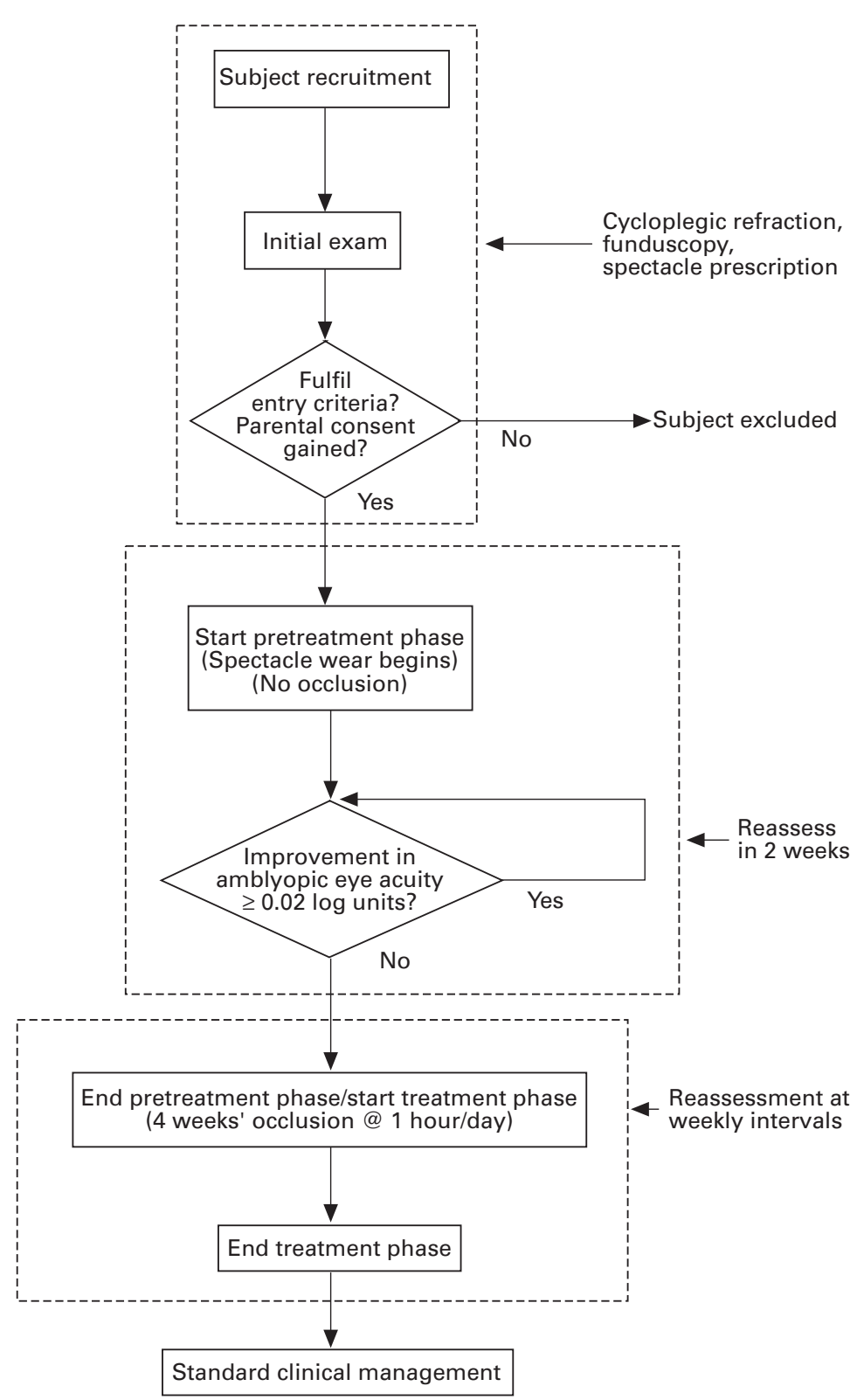

Figure 1 Flow diagram indicating subjects'progression through trial. Boxes (in broken lines) (top to bottom): enrolment, pretreatment phase, treatment phase.

standard deviation of $0.27 \mathrm{log}$ units. The corresponding mean (SD) value of log letter contrast sensitivity was $1.45(0.11)$. In the fellow eyes, mean corrected acuity at this time was $0.25 \log$ MAR (6/11) with SD of $0.22 \mathrm{log}$

Table 1 Subject characteristics at presentation

\begin{tabular}{lllll}
\hline & \multicolumn{3}{l}{ Refraction (dioptres/axis) } \\
\cline { 3 - 5 } Subject & Age (years) & Sex & Right eye & Left eye \\
\hline AJ $^{\star}$ & 4.7 & $\mathrm{M}$ & $+2.00 \mathrm{DS}$ & $+3.25 /+0.75$ at $105^{\circ}$ \\
$\mathrm{ZL}^{\star}$ & 4.9 & $\mathrm{~F}$ & $-1.50 /+1.25$ at $90^{\circ}$ & $-1.50 /-2.50$ at $180^{\circ}$ \\
SM & 9.5 & $\mathrm{M}$ & $+1.00 \mathrm{DS}$ & $+3.00 \mathrm{DS}$ \\
$\mathrm{TL}$ & 4.8 & $\mathrm{~F}$ & $+2.50 /+1.00$ at $20^{\circ}$ & $+3.00 /+1.00$ at $165^{\circ}$ \\
$\mathrm{AC}$ & 5.4 & $\mathrm{~F}$ & Plano & $-11.25 /+1.0$ at $145^{\circ}$ \\
$\mathrm{AI}$ & 5.4 & $\mathrm{M}$ & $-2.50 /-2.00$ at $30^{\circ}$ & Plano \\
DB & 4.3 & $\mathrm{~F}$ & $+5.00 /+1.00$ at $85^{\circ}$ & $+4.50 /+1.50$ at $100^{\circ}$ \\
OS & 3.4 & $\mathrm{~F}$ & $-4.00 /-0.75$ at $75^{\circ}$ & Plano \\
RK & 3.6 & $\mathrm{~F}$ & $+4.50 /+2.00$ at $95^{\circ}$ & Plano \\
JS & 4.5 & $\mathrm{~F}$ & $\infty /+1.75$ at $105^{\circ}$ & $\infty /+1.5$ at $100^{\circ}$ \\
\hline
\end{tabular}

${ }^{\star}$ Did not complete the trial (see text).
Table 2 Percentage concordance with treatment phase occlusion regimen. No objective record available for subject AC during week 1 (see text)

\begin{tabular}{lccccc}
\hline Subject & Week 1 & Week 2 & Week 3 & Week 4 & Average \\
\hline SM & 101 & 102 & 99 & 88 & 98 \\
TL & 116 & 114 & 146 & 133 & 127 \\
AC & - & 86 & 76 & 95 & 86 \\
AI & 104 & 93 & 95 & 89 & 95 \\
DB & 90 & 88 & 82 & 82 & 86 \\
OS & 82 & 63 & 88 & 86 & 80 \\
RK & 66 & 87 & 82 & 92 & 82 \\
JS & 60 & 43 & 45 & 65 & 53 \\
\hline
\end{tabular}

units and with corresponding log contrast sensitivity values of $1.51(0.09)$

Subjects' individually monitored concordance data are shown, on a week by week basis in Table 2. In summary, and with the exception of a single subject (JS), concordance with the trial regimen was judged to be good: all receiving in excess of $80 \%$ of the prescribed dose when averaged over the entire treatment phase. Over the same period the percentage concordance of subject JS averaged only 53\% though at even this level of concordance the accumulated dose was almost 15 hours. The occlusion dose monitors worn by two subjects (TL and AC) suffered episodic failures over a period of 4 and 7 days respectively during which time no objective records could be retrieved. The percentages given in Table 2 have been calculated excluding these occasions although the diaries of these patients (which accurately confirmed the objective records at all other times) suggested that occlusion did take place during the days when the objective record was not available.

Mean visual acuity and letter contrast sensitivity at the points of comparison (initial pretreatment, maximum pretreatment, maximum treatment, final treatment) are shown in Figure 2. The corresponding statistical comparisons are provided in Tables 3 to 5 .

A significant improvement of $0.19 \mathrm{log}$ units $(\mathrm{p}=0.008)$ in acuity and $0.09 \log$ units $(p=0.01)$ in letter contrast sensitivity occurred during pretreatment. Improvements of an all but identical magnitude and significance were also seen in the fellow eye with a gain of 0.19 $\log$ units $(p=0.03)$ in acuity and of $0.11 \log$ units $(p=0.01)$ in letter contrast sensitivity.

A further significant improvement in amblyopic eye visual acuity of $0.12 \log$ units $(p=0.009)$ was apparent when best pretreatment performance was compared with the best that was obtained during any of the 4 weeks of treatment. No corresponding improvement in letter contrast sensitivity occurred in the amblyopic eyes and in the case of the fellow eyes, neither acuity nor letter contrast sensitivity changed significantly during this period.

At the end of treatment the above noted improvement in amblyopic eye acuity seen during treatment was no longer maintainedthat is, there was no significant difference between best pretreatment and end of treatment scores, nor were there any further improvements in acuity of the fellow eye, nor of contrast sensitivity in either eye.

Because of a scheduling confusion, one subject $(\mathrm{RK})$ did not initially enter the treatment 

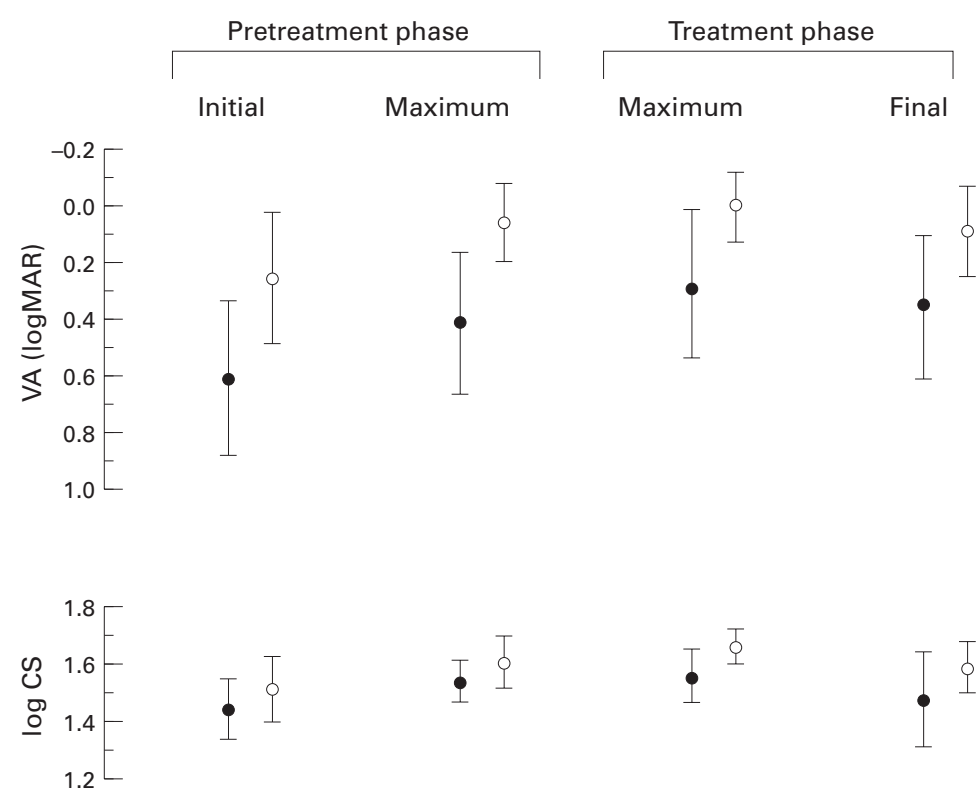

Figure 2 Mean (SD) visual acuity and letter contrast sensitivity of subjects' amblyopic eye (filled circles) and fellow eye (open circles). $N B$ Error bars show variation in scores at the specified points within the trial but do not indicate changes attributable to interventions. The acuity scale has been inverted in order that improvements in visual acuity (VA) and contrast sensitivity (CS) each correspond to vertical increments on the ordinate.

Table 3 Pretreatment phase visual performance (Student's $t$ test): start pretreatment scores versus optimum recorded during this phase. For visual acuity a positive mean difference indicates improvement; for contrast sensitivity improvement is indicated by a negative mean difference

\begin{tabular}{llllll}
\hline $\begin{array}{l}\text { Visual performance } \\
\text { measure }\end{array}$ & Eye & $\begin{array}{l}\text { Mean } \\
\text { difference }\end{array}$ & $S D$ & p Value & $95 \%$ CI \\
\hline Visual acuity & Amblyopic & 0.19 & 0.147 & 0.008 & $(0.07,0.32)$ \\
& Fellow & 0.19 & 0.195 & 0.03 & $(0.03,0.36)$ \\
Contrast sensitivity & Amblyopic & -0.09 & 0.082 & 0.01 & $(-0.16,-0.03)$ \\
& Fellow & -0.11 & 0.105 & 0.02 & $(-0.19,-0.02)$ \\
\hline
\end{tabular}

Table 4 Pretreatment and treatment phase visual performance: optimum scores recorded during pretreatment versus optimum scores recorded during treatment (Student's t test). Improvement/deterioration indicated by sign of mean difference (see Table 3 caption)

\begin{tabular}{llllll}
\hline $\begin{array}{l}\text { Visual performance } \\
\text { measure }\end{array}$ & Eye & $\begin{array}{l}\text { Mean } \\
\text { difference }\end{array}$ & SD & p Value & 95\% CI \\
\hline Visual acuity & Amblyopic & 0.12 & 0.096 & 0.009 & $(0.04,0.20)$ \\
& Fellow & 0.03 & 0.043 & 0.11 & $(-0.01,0.06)$ \\
Contrast sensitivity & Amblyopic & -0.02 & 0.065 & 0.44 & $(-0.07,0.04)$ \\
& Fellow & -0.03 & 0.065 & 0.22 & $(-0.09,0.02)$ \\
\hline
\end{tabular}

Table 5 Pretreatment and treatment phase visual performance: optimum pretreatment scores versus end of treatment scores (Student's $t$ test). Improvement/deterioration indicated by sign of mean difference: (see Table 3 caption)

\begin{tabular}{llclll}
\hline $\begin{array}{l}\text { Visual performance } \\
\text { measure }\end{array}$ & Eye & $\begin{array}{l}\text { Mean } \\
\text { difference }\end{array}$ & $S D$ & $p$ Value & $95 \% C I$ \\
\hline Visual acuity & Amblyopic & 0.06 & 0.086 & 0.09 & $(-0.01,0.13)$ \\
& Fellow & -0.04 & 0.072 & 0.14 & $(-0.01,0.02)$ \\
Contrast sensitivity & Amblyopic & 0.06 & 0.115 & 0.21 & $(-0.04,0.15)$ \\
& Fellow & 0.03 & 0.060 & 0.28 & $(-0.03,0.08)$ \\
\hline
\end{tabular}

phase when her amblyopic eye acuity had stabilised. Interestingly, at the next visit acuity in this eye had begun a second phase of improvement and did not stabilise again until after a further three visits at which time treatment began (Fig 3).

\section{Discussion}

In this pilot study we have examined the practicality of objective concordance monitoring and the need to isolate specific from non- specific treatment effects when evaluating occlusion regimens.

Notwithstanding two instances of failure, the recording of objective concordance data was successfully accomplished. For each subject, we obtained a daily measurement of the occlusion administered and its accumulation throughout the treatment phase. There were no reports of problems encountered in the use of the ODM by the subjects. It did not surprise us that the subjective diary records of concordance were in agreement with the objective records; we made particular efforts in motivating our subjects' parents to complete the diary record and did not involve those for whom we believed this might impose a special burden. It would, however, be extremely unwise to rely on subjective diary records in formal clinical trials of occlusion or indeed in routine clinical practice where an accurate estimate of dose/ concordance is required.

Overall, and perhaps remarkably so, greatest improvements in visual performance occurred during the pretreatment rather than in the treatment phase of the trial. Although spontaneous improvements attributable to spectacle correction have been reported ${ }^{1314}$ the magnitude and pervasiveness (amblyopic eye and fellow eye; visual acuity and contrast sensitivity) of this phenomenon has not been remarked upon in detail. It is unknown if this effect is anti-amblyogenic in a strict physiological sense or arises from perceptual learning ${ }^{18}$ : essentially improvements attributable to practice as the visual system 'learns' to interpret a focused image.

Close inspection of the results, however, reveals that spectacle adaptation cannot be wholly held to account for 'pretreatment effects': four subjects having fellow eye plano lens prescriptions all showed improvement during pretreatment ranging from 0.02 to 0.14 $\log$ units. Though improvement in fellow eyes with non-plano prescriptions was generally greater ( 0.14 to 0.3 log units) it remains significant that an improvement occurred in all fellow eyes with plano correction. It is important to note that these improvements are real and not the 'crests of a wave' of fluctuating performance: in five out of the eight subjects peak performance in fellow eyes was not seen until the final week of pretreatment and in the remaining three subjects, it always occurred in the second half of the pretreatment phase. Relevant to this finding is the observation that subjects' demeanour and receptiveness to undertake the visual tasks tended to improve with time as they became more relaxed within the increasingly familiar environment and with the tester with whom a rapport was steadily developed over the weeks.

In the fellow eye it is of course noteworthy that improvements in performance occurring during pretreatment were seen at all given that all but three subjects had 'unilateral amblyopia'; however with the exception of one subject whose initial pretreatment visual acuity was $0.00 \log$ MAR (6/6) all others had initial pretreatment acuities ranging from 0.08 to $0.34 \log$ MAR (6/7-6/13). Clearly, if the fellow 


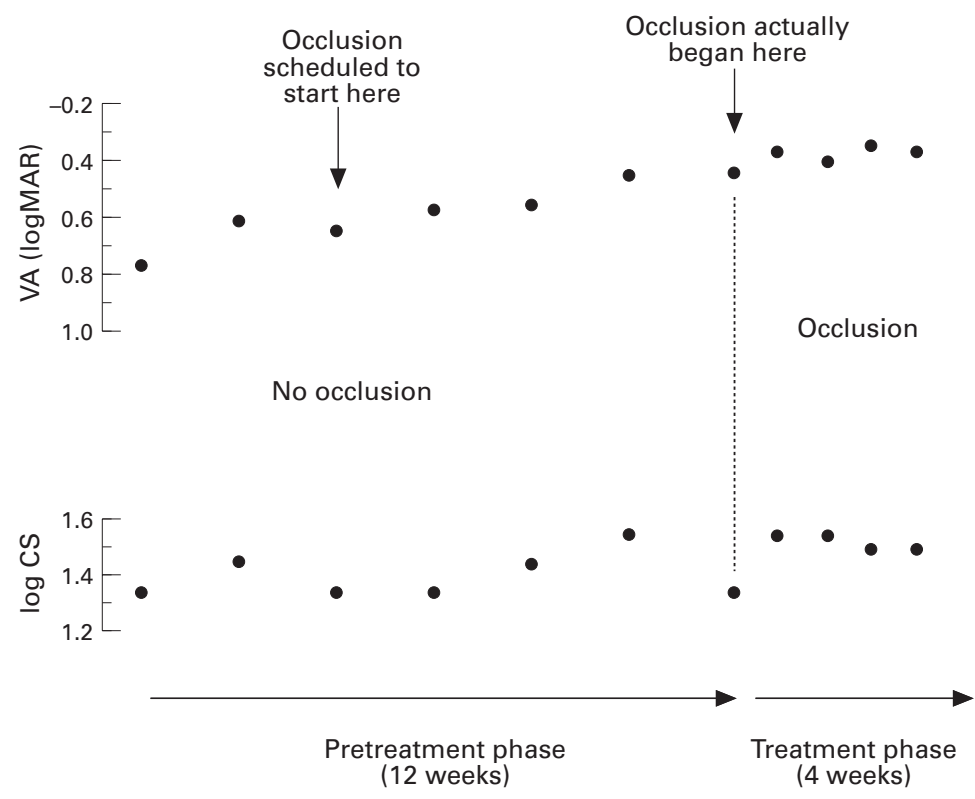

Figure 3 Consecutive performance measures obtained from subject $R K$ during trial. Occlusion was scheduled to begin on the third pretreatment visit (left arrow) but did not in fact commence until 8 weeks later (right arrow). The acuity scale has been inverted in order that improvements in visual acuity (VA) and contrast sensitivity (CS) each correspond to vertical increments on the ordinate.

eye is unaffected in unilateral amblyopia (an assertion increasingly disputed ${ }^{19-21}$ ) it is at least not functionally normal until it has undergone a period of 'pretreatment'. Given the extent of improvement in fellow eye acuity during pretreatment it is unremarkable that further improvements were not seen during the treatment phase as by the end of pretreatment mean acuity of these eyes had reached 0.06 $\log$ MAR (6/7) - that is, not dissimilar from that which might be expected in comparable normal eyes.

In the case of the amblyopic eyes, while a significant improvement of almost two lines in acuity (though not of contrast sensitivity) was seen during treatment, this did not occur in a predictable monotonic fashion-that is, the improvement was not necessarily manifest at the end of treatment with five out of eight subjects obtaining maximum acuity before the end of occlusion. Indeed, by the end of treatment, the gain in acuity over the best obtaining during pretreatment was not statistically significant. At this juncture it is timely to recall that subject RK (who did not enter the treatment phase once her amblyopic eye acuity had initially stabilised, see above) demonstrated a second phase of pretreatment improvement gaining a further $0.20 \mathrm{log}$ units of acuity. We cannot rule out therefore that mechanisms generating improvements during pretreatment were not still operative during the treatment phase. However, these pretreatment effects do not indefinitely continue to benefit visual performance and may to some extent be transitory given that by the end of the treatment phase performance was no better than had previously obtained during pretreatment.

Although a formal statistical power calculation was inapplicable to this pilot study, it is necessary to consider the constraints which insufficient statistical power might impose upon the interpretation of our findings. By definition, where statistical significance was found the necessary power existed. Where, however, significance was not seen (principally in the comparison of best pretreatment with end of treatment scores) lack of power may have led us to falsely accept the null hypothesis of no effect. In other words, had more subjects been recruited then the beneficial effect on acuity (see Table 5) may have attained statistical significance. However, comparing the magnitude of the (statistically significant) improvement occurring during pretreatment with the (statistically insignificant) improvement occurring between pretreatment and end of treatment, the latter is shown to be only one third that of the former: a clinically insignificant three letters. Hence, statistical power considerations do not constrain the conclusion that the effect of pretreatment is clinically significant whereas as that of minimal occlusion is not.

Finally, it is noteworthy to compare the relative sensitivity of the two performance measures in their ability to characterise changes in amblyopic vision. With one exception, where statistically significant changes occurred, acuity and contrast sensitivity were absolutely correlated, although the magnitude of change was, on average, 0.1 log units greater for acuity than for contrast sensitivity (see Table 2). It is therefore unclear what additional information the inclusion of letter contrast sensitivity as a performance outcome measure provides beyond that which can be gained by appropriate measures of visual acuity.

In conclusion, we have investigated two factors-concordance and the separation of specific from non-specific treatment effects, an appreciation of which is vital if the design of future randomised controlled trials of occlusion therapy is to be optimised. It proved possible to record objectively concordance and to derive an individual's occlusion dose. Though not attempted here, such measurements can be equated with concurrent changes in visual performance so as to establish a dose-response relation. We have also shown that careful consideration needs to be given to the isolation of specific treatment (occlusion) effects from other beneficial effects of clinical management found here to be clinically and statistically significant.

\footnotetext{
1 Moseley MJ, Fielder AR. Occlusion therapy for childhood amblyopia: current concepts in treatment evaluation. In: Vital-Durand F, Atkinson J, Braddick OJ, eds. Infant vision Oxford: Oxford University Press, 1996:383-99.

2 von Noorden GK. Binocular vision and ocular motility. 4 th ed. St Louis: C V Mosby, 1990:457-78

3 Watson PG, Sanac AS, Pickering MS. A comparison of various methods of treatment of amblyopia: a block study. Trans Ophthalmol Soc UK 1985;104:319-28.

4 Eggers HM. Amblyopia. In: Podos SM, Yanoff M, eds. Textbook of ophthalmology. Vol 5. Strabismus and pediatric ophthalmology. St Louis: Mosby, 1993:13.2-17.

5 Day S. Normal and abnormal visual development. In: Taylor D, ed. Pediatric ophthalmology. Oxford: Blackwell Scientific Publications, 1990:7-20.

6 Hiscox F, Strong N, Thompson JR, Minshull C, Woodruff G. Occlusion for amblyopia: a comprehensive survey of G. Occlusion for amblyopia:
outcome. Eye 1992;6:300-4.

7 Mullen PD. Compliance becomes concordance. BMf 1997; 314:691-2.
} 
8 Lithander J, Sjöstrand J. Anisometropic and strabismic amblyopia in the age group 2 years and above: a prospective study of th.

9 Leach C. Compliance with occlusion therapy for strabismic and anisometropic amblyopia: a pilot study. Binoc Vis Eye Muscle Surg Qtrly 1995;10:257-66.

10 Oliver M, Neumann R, Chaimovitch Y, Gotesman N, Shimshoni $M$. Compliance and results of treatment for amblyopia in children more than 8 years old. $A m$ f Ophthalmol 1986;102:340-5.

11 Nucci P, Alfarano R, Piantanida A, Brancato R. Compliance in antiamblyopia occlusion therapy. Acta Ophthalmol 1992; 70:128-31

12 Woodruff G, Hiscox F, Thompson JR, Smith LK. Factors affecting the outcome of children treated for amblyopia. Eye 1994;8:627-31.

13 Kivlin JD, Flynn JT. Therapy of anisometropic amblyopia. $\mathcal{F}$ Pediatr Ophthalmol Strabismus 1981;18:47-56.

14 Simons K. Preschool vision screening: rationale, methodology and outcome. Surv Ophthalmol 1996;41:3-30.
15 Fielder AR, Irwin M, Auld R, Cocker KD, Jones HS, Moseley MJ. Compliance in amblyopia therapy: objective monitoring of occlusion. Br f Ophthalmol 1995;79:585-9.

16 Kitchin JE, Bailey I. Task complexity and visual acuity in senile macular degeneration. Aust f Optom 1981;64:23542 .

17 Elliott DB, Bullimore MA, Bailey IL. Improving the reliability of the Pelli-Robson contrast sensitivity test. Clin Vis Sci 1991;6:471-5.

18 Sagi D, Tanne D. Perceptual learning: learning to see. Curr Opin Neurobiol 1994;4:195-9.

19 Grounds AR, Holliday IE, Ruddock KH. Two spatiotemporal filters in human vision. 2 Selective modification in amblyopia, albinism, and hemianopia. Biol Cybernetics 1983;47:191-201.

20 Wali N, Leguire LE, Rogers GL, Bremer DL. CSF interocular interactions in childhood amblyopia. Optom Vis Sci 1991;68:81-7.

21 Woo GC, Irving E. The non-amblyopic eye of a unilateral amblyope: a unique entity. Clin Exp Optom 1991;74:1-5. 